\title{
Personal and Atmospheric Concentrations of Ozone in Southeastern Hyogo Prefecture, Japan
}

\author{
Ning Tang, ${ }^{* a}$ Yoshiko Yoda,${ }^{a}$ Naruhito Otani, ${ }^{a}$ Takayuki Kameda, ${ }^{b}$ Akira Toriba,${ }^{b}$ \\ Kazuichi Hayakawa, ${ }^{b}$ and Masayuki Shima ${ }^{a}$ \\ ${ }^{a}$ Department of Public Health, Hyogo College of Medicine; 1-1 Mukogawa-cho, Nishinomiya 663-8501, Japan: and \\ ${ }^{b}$ Institute of Medical, Pharmaceutical and Health Sciences, Kanazawa University; Kakuma-machi, Kanazawa 920 \\ 1192, Japan. Received February 3, 2012; accepted May 21, 2012
}

Twenty-one data sets composed of readings collected by atmospheric ozone monitors worn by individuals on their clothing and installed outside their home or office were collected using Ogawa passive ozone samplers in southeastern Hyogo prefecture, Japan from September 12 to 13, 2011. The concentrations of personal and outdoor ozone ranged from not detectable to $23.2 \mathrm{ppb}$ and from 4.7 to $38.3 \mathrm{ppb}$, respectively. The mean concentration of personal exposure to ozone was $3.7 \mathrm{ppb}$ and was significantly lower than that of outdoor ozone (18.5 ppb). This suggests that the concentrations of outdoor ozone affect personal ozone exposure. However, in this study, we found no correlation between the concentrations of personal ozone and the total time spent outdoors or the time of day the individual was outside. In contrast, the mean concentrations of outdoor ozone were similar to those of ozone measured at the 12 nearest Ambient Monitoring Stations (AMSs). However, when the AMS was situated near a main road, the regional ozone levels were underestimated.

Key words ozone; air pollution; personal exposure; nitrogen dioxide; Ogawa sampler

Ozone is a strong oxidant and is mainly formed by photochemical reactions from nitrogen oxides and/or reactive volatile organic compounds in the atmosphere. Acute and chronic effects of ozone exposure on human respiratory system have been investigated by many epidemiological studies. ${ }^{1-3)}$ Some researchers have also reported that ozone had negative effects on cardiovascular and respiratory mortality. ${ }^{4-6)}$ A strong relationship has been noted between measured outdoor ozone levels and ambient ozone levels measured by the nearest Ambient Monitoring Station (AMS). ${ }^{7)}$ Ozone concentrations were usually the highest in outdoor and lowest in indoor environment. ${ }^{8,9)}$ Therefore, in the past, these AMS data were sometimes used to evaluate the personal health risk of ozone. ${ }^{5,6}$ However, the specific location of the AMS site and wind direction around the AMS affect its value for evaluating ozone levels at specific localities. The passive ozone sampler, named Harvard or Ogawa passive sampler, developed by Koutrakis et $a l{ }^{10)}$ is a useful tool to accurately measure ozone concentrations in a much smaller area. ${ }^{8,9,11,12)}$ The passive ozone sampler is able to provide a precise measure of the ozone to which a single person may be exposed, which we call the "personal ozone" in this paper. These samplers can measure personal ozone exposure with a precision of $\pm 4 \mathrm{ppb}$ and relative error of $\pm 10 \%{ }^{13}$

In this study, passive ozone samplers were used to evaluate the ozone exposure levels of selected personnel from Hyogo College of Medicine and of their outdoors. This is the first study to determine the ozone exposure levels for people living in Japan. This study also investigates the relationship between personal outdoor ozone exposure levels and the AMS ozone data recorded by the nearest outdoor sampler.

\section{Experimental}

Sampling and Pretreatment Procedures Twenty-one employees of Hyogo College of Medicine were selected as study participants. This study was approved by the ethics

The authors declare no conflict of interest. committee of Hyogo College of Medicine, and informed consent was obtained from each participant before the study. All participants live in five cities of southeastern Hyogo prefecture and their houses are located there (Fig. 1). Each person and house was monitored by a set of personal/outdoor passive ozone samplers (Ogawa sampler manufactured by Ogawa and Co., Ltd., Kobe, Japan). The personal sampler was pinned to the top front side of participant's clothes; the outdoor sampler was placed in a well-ventilated area outside the participant's house. Sampling was performed simultaneously starting at 07:00 September 12, 2011. A total of forty-two 24-h samples were collected and stored in a refrigerator $\left(4^{\circ} \mathrm{C}\right)$ until they were analyzed. The study participants were also instructed to record their daily activities from 7:00 to 12:00, from 12:00 to $18: 00$, and from 18:00 to $24: 00$, so that the amount of time spent outdoors could be estimated (Fig. 2).

The passive ozone sampler consists of two glass fiber filters coated with sodium nitrite and potassium carbonate. ${ }^{14)}$ The mechanism is based on the oxidation reaction of nitrite by ozone to produce nitrate. In this study, the mean concentrations of ozone during the sampling period are estimated by the amount of produced nitrate and collection rate $(21.8 \mathrm{~mL} /$ min). ${ }^{14)}$ The filters were treated according to the Ozone Passive Sampler Protocol published by Ogawa \& Co., Ltd. ${ }^{14)}$ Two glass fiber filters were placed in a vial and $5 \mathrm{~mL}$ ultrapure water (Milli-Q) was poured in the vial. After shaking gently, the vial was left to stand for approximately $30 \mathrm{~min}$. About $1 \mathrm{~mL}$ of supernatant solution was filtered (Cosmonice Filter (W), pore size $0.45 \mu \mathrm{m}, \varphi 13 \mathrm{~mm}$, Nacalai Tesque, Kyoto, Japan) and $100 \mu \mathrm{L}$ of filtrate was injected into the HPLC system described below.

HPLC System and Chemicals Nitrate was analyzed by using an HPLC system with an UV detector. The HPLC system consisted of a mobile phase pump (SI-1/2001, Shiseido, Tokyo, Japan), an UV-visible detector (SI-1/2002, Shiseido, Tokyo, Japan), a chromatopac integrator (C-R7A, Shimadzu, Kyoto, Japan), a degasser (SI-1/2009, Shiseido, Tokyo, Japan), a column oven with a manual injector (SI-1/2004, 


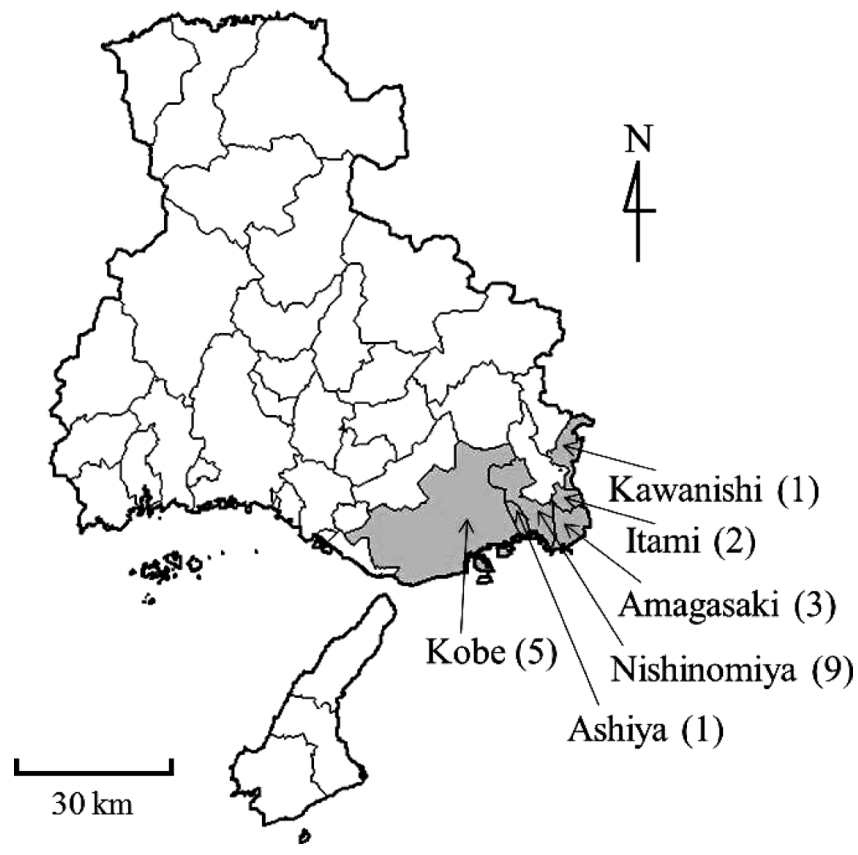

Fig. 1. Cities in Southeastern Hyogo Prefecture in Which Samples Were Collected

Numbers in parentheses indicate the number of samples collected in each city.

Shiseido, Tokyo, Japan), a guard column (Shim-pack ICGA2, 4.6 i.d. $\times 10 \mathrm{~mm}$, Shimadzu, Kyoto, Japan), and a nonsuppressor type analytical column (Shim-pack IC-A3, 4.6 i.d. $\times 150 \mathrm{~mm}$, Shimadzu, Kyoto, Japan). The mobile phase was $0.4 \mathrm{~mm}$ disodium phthalate (Tokyo Kasei, Tokyo, Japan) and the flow rate was $1.0 \mathrm{~mL} / \mathrm{min}$. Both the guard column and the analytical column were maintained at $40^{\circ} \mathrm{C}$ in the column oven. Standard nitrate (sodium nitrate) was purchased from Kishida Chemical Co., Ltd. (Osaka, Japan).

Quality Control Indirect photometric detection ion chromatography was used for nitrate analysis. A standard solution of nitrate $(100 \mu \mathrm{L})$ was injected into the analysis system to verify the method. The calibration curve showed good linearity ( $r=0.9933$ ) from 0.1 to $1.0 \mu \mathrm{g} / \mathrm{mL}$. The detection limit $(S / N=3)$ was $0.1 \mu \mathrm{g} / \mathrm{mL}$ and the relative standard deviation $(n=3)$ was $4 \%$.

In order to assess the accuracy of the passive samplers, data from collocated passive samplers were compared to those obtained from a UV Ozone Monitor (EG-700, EBARAJITSUGYO Co., Ltd., Kanagawa, Japan) at the roof of No. 9 building of Hyogo College of Medicine. The mean ( \pm S.D.) ozone concentrations of five 24-h data sets obtained from passive samplers and Ozone Monitor were $23.5( \pm 2.96) \mathrm{ppb}$ and 23.9 $( \pm 2.14) \mathrm{ppb}$, respectively. In addition, the Ozone Monitorpassive sampler/Ozone Monitor ratio of each data set was less than $10 \%$ (in the range from 0.9 to $8.4 \%$ ). Approximately $10 \%$ of the samplers from the same batch were used as field blanks. The mean $( \pm$ S.D.) concentrations of four field blanks were 11 $( \pm 1.1) \mathrm{ppb}$. The values from the field blanks were subtracted from the ozone measurement for calculation of the concentrations of nitrate collected by the passive samplers.

Data Analysis For statistical treatment, correlation analysis and multiple regression analysis were performed by using a statistical analysis program (PASW Statistic 18, IBM, U.S.A.). For the multiple regression analysis, the following form of the model was used:

$$
C_{\mathrm{o}}=\beta_{1} C_{\mathrm{a}}+\beta_{2} P+\beta_{3} D+\varepsilon
$$

where $C_{\mathrm{o}}$ and $C_{\mathrm{a}}$ are the outdoor and the AMS ozone concentrations, respectively. $P$ is the relative position of the outdoor sampler with respect to the nearest AMS (based on the wind direction including leeward, windward, and parallelism); $D$ is the distance between the outdoor sampler and its nearest AMS and $\varepsilon$ is the error term. The distance between the outdoor samplers and the nearest AMS was between 0.5 and $4.4 \mathrm{~km}$.

\section{Results and Discussion}

Outdoor Ozone A total of 21 outdoor ozone samples were collected for this study. The mean and median ozone concentrations in these sample were 18.5 and $17.0 \mathrm{ppb}$, respectively, the same as the concentration levels recorded from the 12 AMS sites nearest to the outdoor samplers (mean, 16.2ppb; median, 15.5 ppb, Fig. 3). ${ }^{15)}$ However, the concentrations of outdoor ozone measured at study participants' houses ranged from 4.7 to $38.3 \mathrm{ppb}$, while those of ozone measured by the AMS ranged from 9.5 to $20.2 \mathrm{ppb}$. The difference between the two concentration ranges was considerably large (Fig. 3). No clear relationship was observed between the outdoor and AMS ozone concentrations (Fig. 4, solid line; $n=21, r=0.161$, $p=0.481$ ). However, because ozone reacts with nitric oxide in the atmosphere, local variations in nitric oxide concentrations may affect at atmospheric ozone levels. ${ }^{16)}$ When nine data points for the outdoor samplers and the AMS's located within $100 \mathrm{~m}$ of main road were deleted, a significantly positive correlation between the outdoor ozone concentrations and the AMS ozone concentrations exists (Fig. 4, dotted line; $n=12$, $r=0.711, p=0.001)$. Therefore, these results suggest that the ozone concentrations recorded by the AMS, which keeps is placed away from the main road, could represent the mean levels of outdoor ozone concentrations at the home around the AMS.

In order to further evaluate the effects of wind direction and distance on the ozone concentrations determined from outdoor samplers with respect to its nearest AMS, a multiple regression analysis was performed. As shown in Eq. 2, no relationship was observed between the outdoor ozone concentrations and the relative position of the AMS $(p=0.89)$ or the distance $(p=0.11)$.

$$
C_{\mathrm{o}}=42.6-1.02 C_{\mathrm{a}}-0.46 P-3.79 D
$$

Similarly, if the same nine values for the outdoor samplers and the AMS mentioned above were ignored (samples collected within $100 \mathrm{~m}$ of a main road), the analysis result was

$$
C_{\mathrm{o}}=0.26+1.08 C_{\mathrm{a}}-0.80 P-0.58 D
$$

In this model, only the coefficients of outdoor ozone concentrations and the AMS ozone concentrations were significant $(p=0.05)$. The relative position and the distance are less important for the outdoor ozone concentrations measured by the Ogawa samplers.

Personal Ozone This is the first study to report the personal exposure levels of ozone in Japan. A total of 21 personal ozone samples were obtained for this study. The mean and the median concentrations of personal ozone samples were 3.7 and $2.7 \mathrm{ppb}$, respectively, and these concentrations were significantly lower than outdoor ozone (Fig. 3). The range of 


\section{Questionnaire on Ozone Exposure Investigation}

1. ID

2. Address

3. Start Time

$\begin{array}{lllll}\text { ( ) }-1 & \text { Sep. } 122011 & \text { H. } & \text { M. } & \text { (personal) } \\ \text { ( ) }-2 & \text { Sep. } 122011 & \text { H. } & \text { M. } & \text { (outdoor) }\end{array}$

4. Stop Time

$\begin{array}{lllll}\text { ( ) }-1 & \text { Sep. 13 2011 } & \text { H. } & \text { M. } & \text { (personal) } \\ \text { ( ) }-2 & \text { Sep. 13 2012 } & \text { H. } & \text { M. } & \text { (outdoor) }\end{array}$

5. Time in outdoor

$\begin{array}{lll}06: 00-12: 00 & \text { H. } & \text { M. } \\ 12: 00-18: 00 & \text { H. } & \text { M. } \\ 18: 00-24: 00 & \text { H. } & \text { M. }\end{array}$

6. Sampling Method

* Open the container and remove the sampling badge from the resealable bag.

* Check that the ID label on the bottle; (ID)-1 use for personal ozone and (ID)-2 use for outdoor ozone.

* Place sampling badge as same as the following Figures.
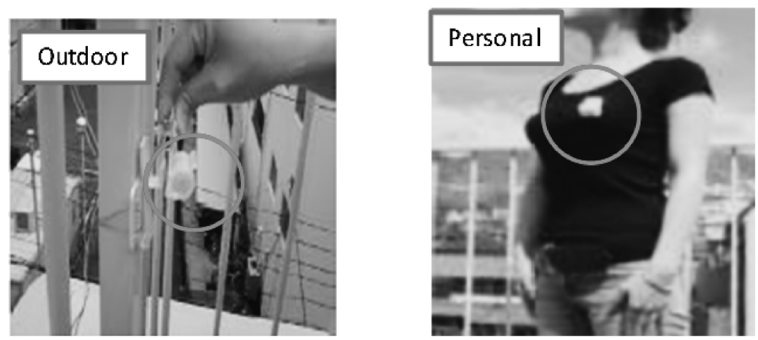

* After sampling, replace the re-sealable bag in the brown storage bottle and tighten cap securely

* Record daily activities during sampling period.

Noto

Thank you very much for your collaboration!

Fig. 2. Questionnaire on Ozone Exposure Investigation

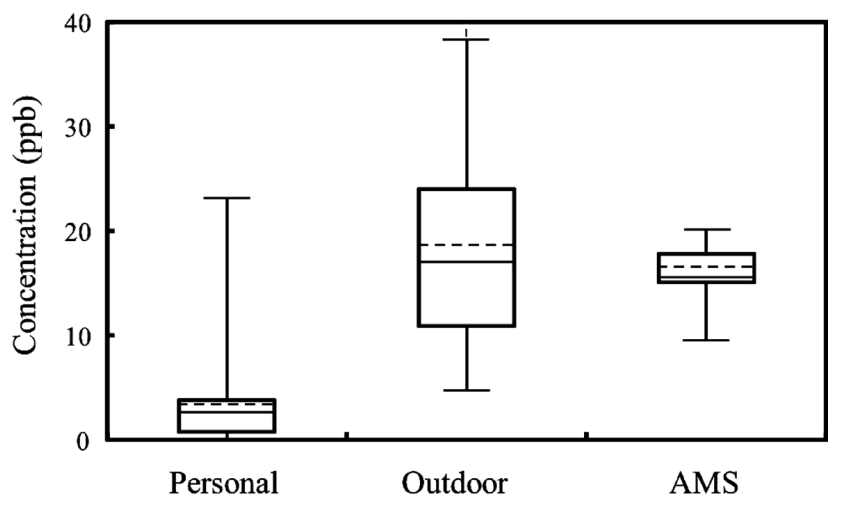

Fig. 3. Ozone Concentrations Found in Personal and Outdoor Samples and Ozone Levels Recorded by the Ambient Monitoring Stations (AMS)

Error bars indicate the range from minimum to maximum concentrations of each sample set. The upper and lower edges of each box represent the 75 th and 25 th percentile concentration ratios, respectively. Within each box, mean and median concentrations are indicated by the dashed and solid lines, respectively.

\section{Department of Public Health, Hyogo College of Medicine}

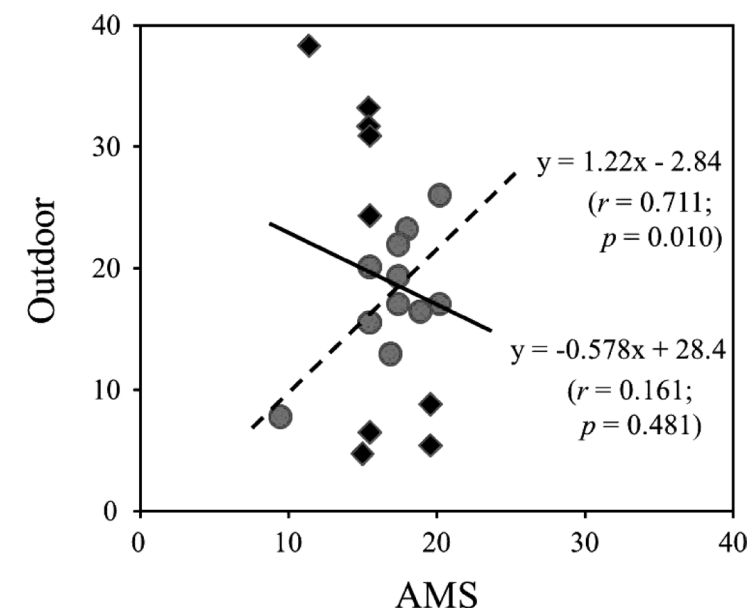

Fig. 4. Correlation between Outdoor Ozone Concentrations and the AMS Ozone Concentrations

Solid line: all data $(n=21)$, Dotted line: nine data points for the outdoor samplers and the AMS's located within $100 \mathrm{~m}$ of main road were deleted $(n=12)$ 


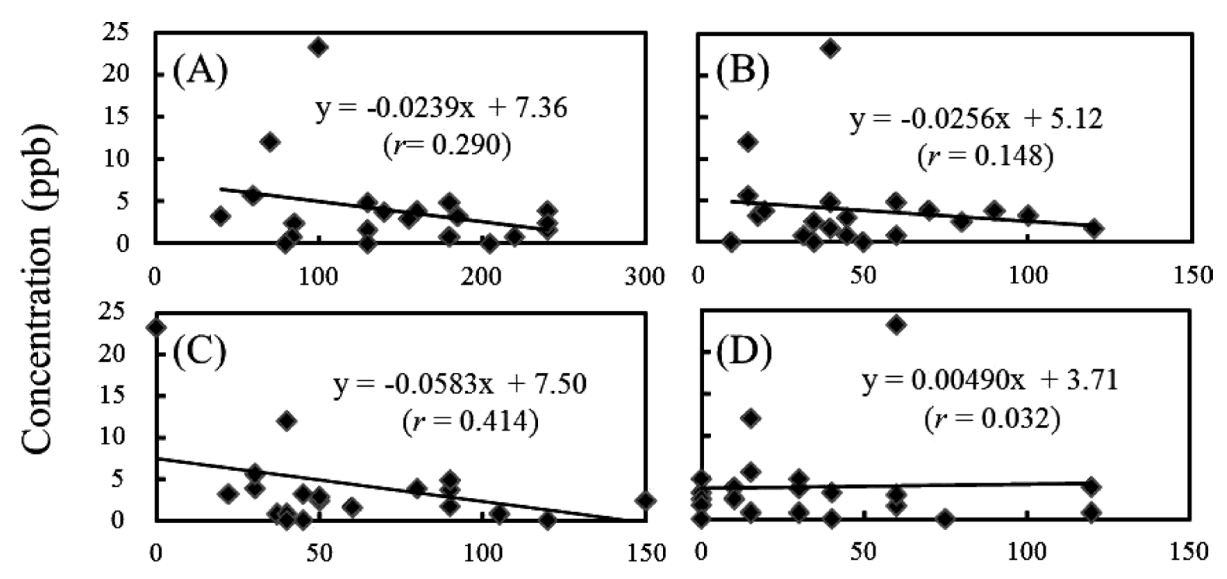

\section{Time (min)}

Fig. 5. Distribution of Data Points Showing the Lack of Correlation between Personal Ozone Concentrations and the Total Amount of Time Spent Outdoors

(A) 07:00-24:00; (B) 07:00-12:00; (C) 12:00-18:00; (D) 18:00-24:00.

concentrations for personal ozone was from not detectible to $23.2 \mathrm{ppb}$. Compared with other studies using the same sampling methodology, the mean concentrations of personal ozone found in this study were similar to levels as in Nashville $(3.5 \mathrm{ppb})^{9)}$ and lower than those in Mexico City $(7.8 \mathrm{ppb}){ }^{16)}$

Atmospheric ozone is formed during daylight from complex mixtures of nitrogen oxides and reactive volatile organic compounds by photochemical reactions. ${ }^{17)}$ At night, however, ozone reacts with nitrogen monoxide and disappears slowly. ${ }^{18}$ Therefore, ozone concentrations are usually the highest in the afternoon and low in the morning. Lee et al. reported that the concentrations of personal ozone were affected not only by the total amount of time spent outdoors but also the time of day the personal was outside.9) In this study, we plotted the concentrations of personal ozone and personal daily activities, as shown in Figs. 5A through D. We, however, found no correlation between the concentrations of personal ozone and the total time spent outdoors or the time of day the individual was outside. Furthermore, we also found no correlation between the concentrations of personal ozone and the concentrations of outdoor ozone at the same locality $(r=-0.208, p=0.352)$. We cannot explain these results, perhaps because our questionnaire did not include questions about the office or house construction, equipment, and outdoor environment that they spent during sampling period. Items such as a window fan, ${ }^{9)}$ a laser printer, ${ }^{19)}$ or an air cleaner ${ }^{20)}$ might increase ozone levels, and the close proximity of a main road might be decrease the ozone level.9) An improved questionnaire will be used in our next study.

In conclusion, the concentrations of personal ozone and outdoor ozone in southeastern Hyogo were investigated by using the Ogawa sampler. The concentrations of personal ozone were significantly lower than that of outdoor ozone. This suggests that the concentrations of outdoor ozone are important for personal ozone exposure. However, our data found no correlation between the concentrations of personal ozone and the total time spent outdoors or the time of day the individual was outside. In contrast, according to our data, the regional ozone levels may be underestimating by installation feature of the AMS, such as near the main roads.
Acknowledgments This work was supported in part by the Grants-in-Aid for Scientific Research (Nos. 23249034, 21256001, and 21590132) from the Ministry of Education, Culture, Sports, Science and Technology of Japan. The authors thank the staff of the Center of Hyogo Unit of the Japan Environment and Children's Study for participating in this study.

\section{References}

1) Spektor D. M., Thurston G. D., Mao J., He D., Hayes C., Lippmann M., Environ. Res., 55, 107-122 (1991).

2) Diette G. B., Hansel N. N., Buckley T. J., Curtin-Brosnan J., Eggleston P. A., Matsui E. C., McCormack M. C., Williams D. L., Breysse P. N., Environ. Health Perspect., 115, 1665-1669 (2007).

3) Khatri S. B., Holguin F. C., Ryan P. B., Mannino D., Erzurum S. C., Teague W. G., J. Asthma, 46, 777-785 (2009).

4) Wong C. M., Ma S., Hedley A. J., Lam T. H., Environ. Health Perspect., 109, 335-340 (2001).

5) Lipfert F. W., Morris S. C., Wyzga R. E., J. Air Waste Manage. Assoc., 50, 1501-1513 (2000).

6) Ren C., Melly S., Schwartz J., Environ. Health, 9, 3 (1-10) (2010).

7) O'Neill M. S., Ramirez-Aguilar M., Meneses-Gonzalez F., Hernandez-Avila M., Geyh A. S., Sienra-Monge J. J., Romieu I., J. Air Waste Manage. Assoc., 53, 339-346 (2003).

8) Liu L. J. S., Koutrakis P., Leech J., Broder I., J. Air Waste Manage. Assoc., 45, 223-234 (1995).

9) Lee K., Parkhurst W. J., Xue J., Ozaynak H., Neuberg D., Spengler J. D., J. Air Waste Manage. Assoc., 54, 352-359 (2004).

10) Koutrakis P., Wolfson J. M., Bunyaviroch A., Froehlich S. E., Hirano K., Mulik J. D., Anal. Chem., 65, 209-214 (1993).

11) Geyh A. S., Xue J., Ozkaynak H., Spengler J. D., Environ. Health Perspect., 108, 265-270 (2000).

12) Geyh A. S., Roberts P. T., Lurmann F. W., Schoell B. M., Avol E. L., J. Expo. Anal. Environ. Epidemiol., 9, 143-149 (1999).

13) Liu L. J. S., Olson M. P., Allen G. A., Koutrakis P., McDonnell W. F., Gerrity T. R., Environ. Sci. Technol., 28, 915-923 (1994).

14) Ogawa \& Co., U.S.A., Inc., "Protocol for ozone measurement using the ozone passive sampler badge": 〈http://ogawausa.com/〉, Rev. 3, February, 2001.

15) Ministry of the Environment Government of Japan, "Atmospheric Environmental Regional Observation System”: 〈http://soramame. taiki.go.jp/Index.php). cited September 12-13, 2011.

16) Ramírez-Aguilar M., Barraza-Villarreal A., Moreno-Macías H., Winer A. M., Cicero-Fernández P., Vélez-Márquez M. G. D., 
Cortez-Lugo M., Sienra-Monge J. J., Romieu I., Salud Publica Mex., 50, 67-75 (2008).

17) Demerjian K. L., Kerr J. A., Calvert J. G., "Advances in Environmental Science and Technology," Vol. 4, ed. by Pitts J. N., Metcalf R. L., Wiley-Interscience, 1974.

18) Tucker A. W., Birnbaum M., Fincher C. L., Appl. Opt., 14,
1418-1422 (1975).

19) Tang T., Gminski R., Konczol M., Modest C., Armbruster B., Mersch-Sundermann V., Environ. Mol. Mutagen., 53, 125-135 (2012).

20) Tung T. C. W., Niu J. L., Burnett J., Hung K., Indoor Built Environ., 14, 29-37 (2005). 